\title{
Advantages and disadvantages of F-18 fluorodeoxyglucose positron emission tomography/computed tomography in carcinoma of unknown primary
}

\author{
XIAOZHOU YU ${ }^{1-3}$, XIAOFENG LI ${ }^{1-3}$, XIUYU SONG $^{1-3}$, DONG DAI ${ }^{1-3}$, LEI ZHU $^{1-3}$, \\ YANJIA ZHU ${ }^{1-3}$, JIAN WANG ${ }^{1-3}$, HUIQIN ZHAO ${ }^{1-3}$ and WENGUI XU ${ }^{1-3}$ \\ ${ }^{1}$ Department of Molecular Imaging and Nuclear Medicine, Tianjin Medical University Cancer Institute and Hospital; \\ ${ }^{2}$ National Clinical Research Center for Cancer; ${ }^{3}$ Key Laboratory of Cancer Prevention and Therapy, Tianjin 300060, P.R. China
}

Received July 8, 2015; Accepted September 12, 2016

DOI: $10.3892 / 01.2016 .5203$

\begin{abstract}
Carcinoma of unknown primary is a type of malignant disease where the primary carcinoma cannot be identified by conventional examination, which presents challenges in diagnosis and therapy. This study aims to evaluate the detailed clinical value and indications of using fluorine-18-2-fluoro-2-deoxy-D-glucose positron emission tomography/computed tomography (F-18 FDG PET/CT) in a large sample. A total of 449 patients who were selected under strict standards were retrospectively included in this study. F-18 FDG PET/CT accurately detected the primary carcinoma in 115 of 449 patients whose primaries could not be detected by conventional examination $(25.6 \%)$, with additional 27 false-positive patients. The most common primary site was the lung (34.8\%). In addition, except for in metastatic melanoma $(1 / 19,5.3 \%)$ and axillary metastasis patients $(2 / 49$, 4.1\%), F-18 FDG PET/CT had a comparative performance in detecting primary carcinoma in other pathological types and anatomical locations. The scan is able to guide treatment strategy modifications to some extent (130/449, 29.0\%). We strongly recommend the use of F-18 FDG PET/CT in the early phase of examination. It is also recommended as a supplementary radiological method, and certain patients may benefit from its application in cases where regular examination
\end{abstract}

Correspondence to: Dr Wengui Xu, Department of Molecular Imaging and Nuclear Medicine, Tianjin Medical University Cancer Institute and Hospital, Huanhuxi Road, Ti-Yuan-Bei, Hexi, Tianjin 300060, P.R. China

E-mail:wxu06@tmu.edu.cn

Abbreviations: CUP, carcinoma of unknown primary; F-18 FDG PET/CT, fluorine-18-2-fluoro-2-deoxy-D-glucose positron emission tomography/computed tomography; MRI, magnetic resonance imaging; BGC, blood glucose concentration; $\mathrm{mCi}$, millicurie; $\mathrm{LN}$, lymph node

Key words: carcinoma of unknown primary, F-18 FDG PET/CT, melanoma, adenocarcinoma, squamous cell carcinoma is inconclusive. However, in metastatic melanoma or axillary metastasis patients where the primary site cannot be identified by routine examination, regular application of F-18 FDG PET/CT for the sole purpose of detecting the primary carcinoma should not be encouraged.

\section{Introduction}

Carcinoma of unknown primary (CUP) is generally defined as a category of histologically proven malignancy with a primary tumor that cannot be detected by conventional diagnostic methods (1). As a type of heterogeneous disease, it accounts for $0.5-9 \%$ of all cases of malignant diseases (2). Since the primary cannot be identified effectively, most patients receive empirical therapy in clinical practice with unsatisfactory outcome $(3,4)$. Under these circumstances, although certain individuals with favorable prognoses benefit from therapy, the majority of such patients generally have poor outcome with a median life expectancy of less than 12 months $(5,6)$.

Fluorine-18-2-fluoro-2-deoxy-D-glucose positron emission tomography/computed tomography (F-18 FDG PET/CT) has high sensitivity in detecting multiple malignancies, and is routinely applied clinically for staging, restaging and monitoring treatment (7-9). In addition, a number of studies have indicated the crucial role of F-18 FDG PET/CT in CUP patients (10-13). Generally, the previous studies indicated that F-18 FDG PET/CT was able to detect the primary (9.6-47.2\%) and guide the choice of treatment (29.4-47\%) in approximately one-third of all patients $(12,14-18)$. Although these studies provided useful evidence to guide clinical practice, their limitations were still significant, and further improvement is required. First of all, the definition of CUP was not unified and specific in previous studies. Secondly, certain studies did not exclude lymphoma and hematological malignant disease from the samples, which may have led to an underestimation of the actual detection rate. In addition, not all patients in the previous studies received biopsy to prove the metastases, or extensive conventional examination to identify the primaries. Furthermore, the most relevant studies had a small sample size that was generally no larger than 200 individuals. Finally, this issue was seldom investigated with respect to 
Table I. Pathological type and localization of metastases.

\begin{tabular}{|c|c|c|c|c|c|c|c|}
\hline & $\begin{array}{l}\text { Pathological } \\
\text { type }\end{array}$ & Adenocarcinoma & $\begin{array}{c}\text { Squamous } \\
\text { cell carcinoma }\end{array}$ & $\begin{array}{l}\text { Small-cell } \\
\text { carcinoma }\end{array}$ & Melanoma & Other ${ }^{\mathrm{c}}$ & Undefined \\
\hline $\begin{array}{l}\text { Localization of } \\
\text { metastases }\end{array}$ & Total $(n=449)$ & 179 (39.9) & $121(26.9)$ & $26(5.8)$ & $19(4.2)$ & $28(6.2)$ & $76(16.9)$ \\
\hline $\mathrm{LN}$ or soft tissue & $338(75.2)$ & $122(31.6)$ & $113(25.2)$ & $26(5.8)$ & $16(3.6)$ & $20(4.4)$ & $41(9.1)$ \\
\hline Cervical & 169 (37.6) & $37(8.2)$ & 89 (19.8) & $10(2.2)$ & $5(1.1)$ & $6(1.3)$ & $22(4.9)$ \\
\hline Supraclavicular & $58(12.9)$ & $26(5.8)$ & $16(3.6)$ & $6(1.3)$ & $0(0.0)$ & $4(0.9)$ & $6(1.3)$ \\
\hline Axillary & $49(10.9)$ & $35(7.8)$ & $0(0.0)$ & $2(0.4)$ & $6(1.3)$ & $1(0.2)$ & $5(1.1)$ \\
\hline Inguinal & $28(6.2)$ & $8(1.8)$ & $5(1.1)$ & $2(0.4)$ & $5(1.1)$ & $4(0.9)$ & $4(0.9)$ \\
\hline Mediastinal & $12(2.7)$ & $3(0.7)$ & $2(0.4)$ & $5(1.1)$ & $0(0.0)$ & $1(0.2)$ & $1(0.2)$ \\
\hline Retroperitoneal & $10(2.2)$ & $6(1.3)$ & $0(0.0)$ & $0(0.0)$ & $0(0.0)$ & $2(0.4)$ & $2(0.4)$ \\
\hline Abdominal/pelvic & $3(0.7)$ & $2(0.4)$ & $0(0.0)$ & $0(0.0)$ & $0(0.0)$ & $0(0.0)$ & $1(0.2)$ \\
\hline Iliac & $1(0.2)$ & $0(0.0)$ & $1(0.2)$ & $0(0.0)$ & $0(0.0)$ & $0(0.0)$ & $0(0.0)$ \\
\hline Other ${ }^{\mathrm{a}}$ & $8(1.8)$ & $5(1.1)$ & $0(0.0)$ & $1(0.2)$ & $0(0.0)$ & $2(0.4)$ & $0(0.0)$ \\
\hline Skeleton & $49(10.9)$ & $26(5.8)$ & $3(0.7)$ & $0(0.0)$ & $0(0.0)$ & $3(0.7)$ & $17(3.8)$ \\
\hline Axial & $36(8.0)$ & $19(4.2)$ & $1(0.2)$ & $0(0.0)$ & $0(0.0)$ & $2(0.4)$ & $14(3.1)$ \\
\hline Other & $13(2.9)$ & 7 (1.6) & $2(0.4)$ & $0(0.0)$ & $0(0.0)$ & $1(0.2)$ & $3(0.7)$ \\
\hline Brain & $22(4.9)$ & $9(2.0)$ & $0(0.0)$ & $0(0.0)$ & $2(0.4)$ & $3(0.7)$ & $8(1.8)$ \\
\hline Liver & $15(3.3)$ & $9(2.0)$ & $1(0.2)$ & $0(0.0)$ & $0(0.0)$ & $2(0.4)$ & $3(0.7)$ \\
\hline Gland & $7(1.5)$ & $1(0.2)$ & $4(0.9)$ & $0(0.0)$ & $1(0.2)$ & $0(0.0)$ & $1(0.2)$ \\
\hline Parotid & $5(1.1)$ & $0(0.0)$ & $4(0.9)$ & $0(0.0)$ & $1(0.2)$ & $0(0.0)$ & $0(0.0)$ \\
\hline Adrenal & $2(0.4)$ & $1(0.2)$ & $0(0.0)$ & $0(0.0)$ & $0(0.0)$ & $0(0.0)$ & $1(0.2)$ \\
\hline Effusion & $6(1.3)$ & $5(1.1)$ & $0(0.0)$ & $0(0.0)$ & $0(0.0)$ & $0(0.0)$ & $1(0.2)$ \\
\hline Pleural & $3(0.7)$ & $3(0.7)$ & $0(0.0)$ & $0(0.0)$ & $0(0.0)$ & $0(0.0)$ & $0(0.0)$ \\
\hline Pericardiac & $2(0.4)$ & $2(0.4)$ & $0(0.0)$ & $0(0.0)$ & $0(0.0)$ & $0(0.0)$ & $0(0.0)$ \\
\hline Ascitic & $1(0.2)$ & $0(0.0)$ & $0(0.0)$ & $0(0.0)$ & $0(0.0)$ & $0(0.0)$ & $1(0.2)$ \\
\hline Lung & $6(1.3)$ & $2(0.4)$ & $0(0.0)$ & $0(0.0)$ & $0(0.0)$ & $0(0.0)$ & $4(0.9)$ \\
\hline Pleural membrane & $2(0.4)$ & $2(0.4)$ & $0(0.0)$ & $0(0.0)$ & $0(0.0)$ & $0(0.0)$ & $0(0.0)$ \\
\hline Other $^{\mathrm{b}}$ & $4(0.9)$ & $3(0.7)$ & $0(0.0)$ & $0(0.0)$ & $0(0.0)$ & $0(0.0)$ & $1(0.2)$ \\
\hline
\end{tabular}

All patients received biopsy at the initially presented localization of metastases. Clinicopathological characteristics were collected. ${ }^{\mathrm{a} T h e}$ 'LN or soft tissue - Other' group contained 7 patients with metastases from the thoracic or abdominal wall and 1 patient with metastases from the vagina. ' ${ }^{\text {The }}$ 'Other' localization of metastases group contained 2 patients with metastases from the ovary, 1 patient with metastases from the bladder and 1 patient with metastases from the eyes. 'Other pathological types included 4 cases of large-cell carcinoma, 3 cases of sarcoma, 7 cases of clear cell carcinoma, 1 case of hepatocellular carcinoma, 1 case of mesenchymoma, 7 cases of papillocarcinoma and 5 cases of mixed carcinoma. LN, lymph node.

different pathological types and locations. This study aimed to investigate the value and indications of F-18 FDG PET/CT in CUP patients. Utilizing a large sample size, we focused on identifying the primary and the role of the scan in guiding treatment plans according to the different metastatic sites and pathological types.

\section{Materials and methods}

Patients. The present study was approved by the ethics committee of Tianjin Cancer Institute and Hospital, China, and conducted in accordance with the Declaration of Helsinki. Written informed consent was obtained from the patientsor their family. In this retrospective study, selected cases were consecutively included among 26,763 patients who received an F-18 FDG PET/CT scan between January 2006 and October 2014 in Tianjin Medical University Cancer Institute and Hospital, China. All individuals had biopsy-proven malignant metastases. However, prior to receiving the F-18 FDG PET/CT scan, the primary could not be confirmed using regular methods, including detailed physical examination, routine serum tumor marker test and other imaging auxiliary examinations including chest X-ray, CT, magnetic resonance imaging (MRI), mammography (in females), cervical, abdominal and breast (in females) ultrasonography and endoscopy. The biopsy samples of all patients were stained using immunohistochemistry methods to exclude hematological malignant disease or lymphoma, and to preliminarily predict the primary location. Following the F-18 FDG PET/CT scan, the potential primary carcinoma was routinely confirmed by 
biopsy or imaging follow-up. The minimum follow-up period was 9 months, and patients who did not attend a follow-up appointment were excluded from the study. Finally, a total of 449 patients were included.

Imaging. The F-18 FDG PET/CT scan was performed using a Discovery ST PET/CT scanner or a Discovery PET/CT 710 scanner (GE Healthcare, Milwaukee, WI, USA). All patients fasted for at least $6 \mathrm{~h}$, and the blood glucose concentration (BGC) of each patient was monitored prior to intravenous injection of F-18 FDG. For those poorly controlled diabetic patients with high BGC, 4-12 international units of fast-acting insulin were intravenously injected prior to tracer administration. The injection dose of radioactive tracer was calculated as 0.11-0.13 millicuries per kilogram of body weight. The F-18 FDG PET/CT scan was conducted according to a standard protocol. A whole-body scan from mid-thigh to vertex commenced at $\sim 60$ min after injection. Non-contrast-enhanced CT was conducted with a current of 120-170 mA, a voltage of $120 \mathrm{kV}$, a section thickness of 5 or $3.75 \mathrm{~mm}$ and a reconstruction interval of 5 or $3.75 \mathrm{~mm}$. The attenuation-corrected PET image was scanned at 2 min per frame and reconstructed using CT data with iterative algorithms.

Image interpretation. Morphological, metabolic and fused PET/CT images were inspected in axial, coronal and sagittal view using Xeleris software from GE Healthcare. Three senior nuclear medicine physicians independently interpreted the PET/CT images (based on clinical pathological data, location, shape, CT attenuation and F-18 FDG uptake), and then reached a consensus to the diagnosis of all patients.

Statistical analysis. Categorical variables were recorded as numbers and percentages. The median and range were used to express abnormal distribution variables. All statistics were analyzed using SPSS 17.0 software (IBM SPSS, Armonk, NY, USA).

\section{Results}

Metastases and primary sites. The median age of patients was 58 years old (range, 13-83 years). A total of 261 male cases (58.1\%) and 188 female patients $(41.9 \%)$ were included in this study. The pathological type and localization of metastases are summarized in Table I.

Using F-18 FDG PET/CT, the primary sites of 115 cases were located (115 of 449, 25.6\%) (Table II). Representative cases are shown in Fig. 1. Of these patients, 40 primaries were identified in the lung (34.8\%), 16 in the nasopharynx (13.9\%), 13 in the pancreas (11.3\%), 11 in the tonsil (9.6\%), 8 in the small intestine (7.0\%), 6 in the stomach (5.2\%), 4 in the larynx (3.5\%), 4 in the ovary $(3.5 \%), 3$ in the esophagus $(2.6 \%), 3$ in the colorectum (2.6\%), 2 in the kidney $(1.7 \%), 1$ in the male mammary gland $(0.9 \%), 1$ in the prostate $(0.9 \%), 1$ in the penis $(0.9 \%), 1$ in the cholecyst $(0.9 \%)$ and 1 in the uterus $(0.9 \%)$. In addition to the previously known metastases, F-18 FDG PET/CT identified additional metastatic foci in 131 of the 449 patients (29.2\%).

Pathologically, F-18 FDG PET/CT identified primary carcinoma in 29 of the 76 patients with undefined pathological type (38.2\%), 36 of 121 squamous cell carcinoma patients
Table II. Localization of primary sites that were accurately identified by fluorine-18-2-fluoro-2-deoxy-D-glucose positron emission tomography/computed tomography in 115 patients.

\begin{tabular}{lc}
\hline Localization of primaries & No. of patients $(\%)$ \\
\hline Lung & $40(34.8)$ \\
Nasopharynx & $16(13.9)$ \\
Pancreas & $13(11.3)$ \\
Tonsil & $11(9.6)$ \\
Small intestine & $8(7.0)$ \\
Stomach & $6(5.2)$ \\
Larynx & $4(3.5)$ \\
Ovary & $4(3.5)$ \\
Esophagus & $3(2.6)$ \\
Colorectum & $3(2.6)$ \\
Kidney & $2(1.7)$ \\
Male mammary gland & $1(0.9)$ \\
Prostate & $1(0.9)$ \\
Penis & $1(0.9)$ \\
Cholecyst & $1(0.9)$ \\
Uterus & $1(0.9)$ \\
\hline
\end{tabular}

(29.8\%), 8 of 28 other pathological type patients (28.6\%), 38 of 179 adenocarcinoma patients $(21.2 \%), 3$ of 26 small-cell carcinoma patients $(11.5 \%)$ and 1 of 19 melanoma patients $(5.3 \%)$ (Table III).

We also analyzed the efficiency of F-18 FDG PET/CT in detecting the primary carcinoma according to different metastatic sites (sample size more than 20). Metastases initially identified in the head and neck (cervical and supraclavicular metastasis) accounted for the majority of patients (227 of 449, $50.6 \%$ ). F-18 FDG PET/CT identified primary carcinoma in 66 of 227 patients $(29.1 \%)$. Of these, 20 primaries were located in the lung (30.3\%), 16 in the nasopharynx (24.2\%), 11 in the tonsil $(16.7 \%), 4$ in the pancreas $(6.0 \%), 3$ in the stomach $(4.5 \%)$, 3 in the larynx (4.5\%), 2 in the small intestine (3.0\%), 2 in the esophagus (3.0\%), 1 in the ovary (1.5\%), 1 in the colorectum (1.5\%), 1 in the kidney (1.5\%), 1 in the male mammary gland $(1.5 \%)$ and 1 in the cholecyst (1.5\%). Among the 49 patients who were initially identified as having axillary metastases, the primaries were determined by F-18 FDG PET/CT in just 2 patients (pancreas and small intestine; 2/49, 4.1\%). In addition, F-18 FDG PET/CT successfully identified the primary sites in 19 of 49 osseous metastasis patients (38.8\%), 5 of 28 inguinal metastasis patients $(17.9 \%)$ and 5 of 22 cerebral metastasis patients $(22.7 \%)$ (Table IV).

F-18 FDG PET/CT guided treatment change. As a result of the F-18 FDG PET/CT scan, the treatment plans of 130 of the 449 patients $(20.9 \%)$ required modification. Fourteen patients canceled surgery due to upstage or extra metastases being identified by F-18 FDG PET/CT (3.1\%). A total of 121 patients initiated or modified systematic treatment plans guided by F-18 FDG PET/CT scan, including chemotherapy and immunotherapy $(26.9 \%)$. Among these, although without identification of their primary carcinoma, 11 patients changed their chemotherapy 
A
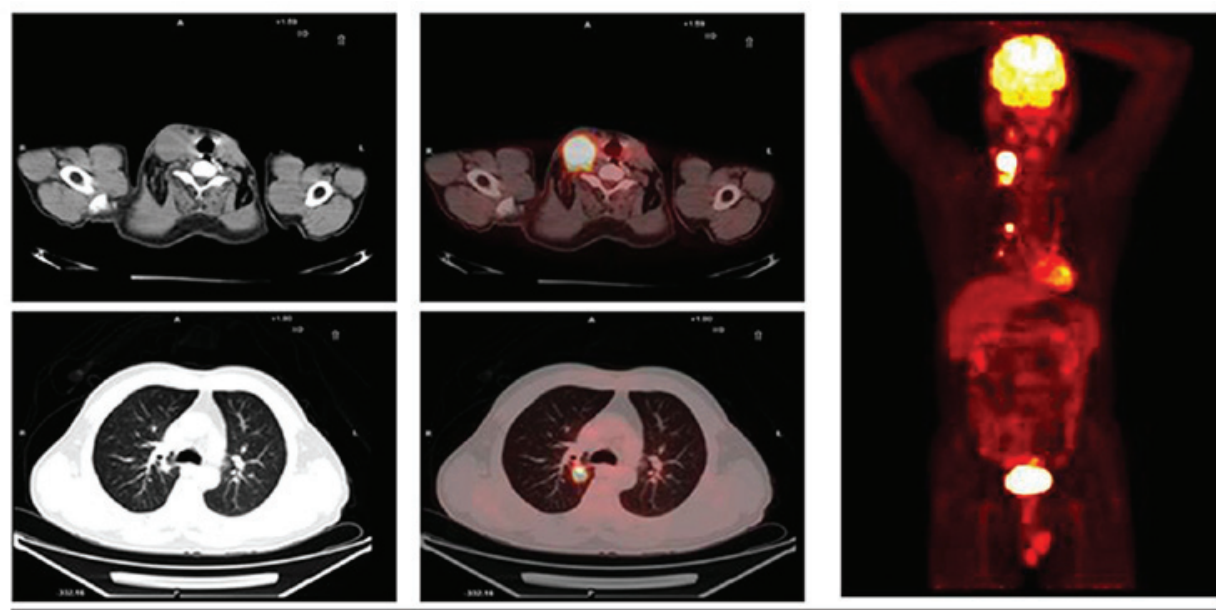

B
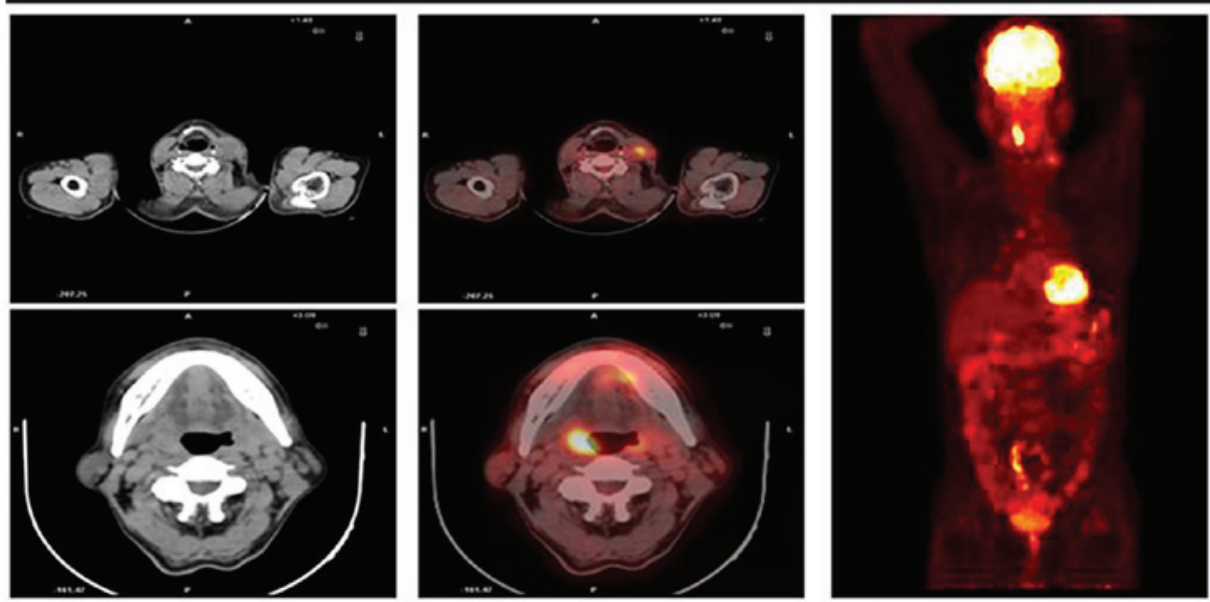

Figure 1. Representative imaging from F-18 FDG PET/CT scans in carcinoma of unknown primary patients. (A) A 69-year-old male patient presented with swelling of the right cervical lymph nodes. Biopsy proved metastasis with a pathological type of squamous cell carcinoma. F-18 FDG PET/CT scan identified increased uptake of radioactive tracer in the lung (SUVmax 10.1). Biopsy finally confirmed the primary site as the lung. (B) A 66-year-old male patient initially presented with left cervical lymph node metastasis (squamous cell carcinoma proven by biopsy). F-18 FDG PET/CT located the primary site as the right tonsil (SUVmax 11.4). Biopsy confirmed the diagnosis. Surgical treatment was canceled, and the patients received systemic chemotherapy. F-18 FDG PET/CT, fluorine-18-2-fluoro-2-deoxy-D-glucose positron emission tomography/computed tomography; SUV, standardized uptake value.

and immunotherapy plan due to the extra metastases and focal distribution identified by F-18 FDG PET/CT (2.4\%).

Among the 227 patients with metastases initially identified in the head and neck, 73 changed their treatment strategy following the F-18 FDG PET/CT scan (32.2\%). Of these patients, 6 canceled their surgical treatment $(2.6 \%)$ and 69 modified their chemotherapy and immunotherapy strategy $(30.4 \%)$.

False positive identification of primary carcinoma. Other than the 115 patients whose primaries were correctly identified, the primaries of 27 patients were incorrectly identified, as proven by biopsy or follow-up. Of these patients, pathological biopsy indicated that 9 cases had benign lesions in the gastrointestinal tract, 3 patients in the thyroid, 1 patient in the mammary gland, 7 patients in the head and neck, and 4 patients in the lung. The primaries of the other 3 patients could not be confirmed by follow-up.

\section{Discussion}

At present, CUP is generally accepted as a type of malignant disease which differs from other metastatic malignant carcinomas in that the primary tumor site is not known. Accurate identification of the primary site and precise staging enhance the prognosis significantly (19).

Previous studies performed exploratory work on the use of F-18 FDG PET/CT in CUP and gained instructive conclusions. However, in certain aspects, there is still a long way to go. For instance, Pelosi et al are considered to be the first team that focused on this topic back in 2006 (20). Although their study innovatively evaluated the application of F-18 FDG PET/CT in CUP patients, with the small sample size of 68 , it would be difficult to avoid bias. In 2008, a meta-analysis containing a relatively large sample of 430 patients further discussed this issue (21). However, a series of confounding factors, including the definition of CUP, epidemiology, diagnostic level, types of auxiliary examination, imaging interpretation and enrollment standards may hamper the reliability of this study. In 2013, Wang et al analyzed a large sample containing 164 patients in China (12). However, the study included patients without biopsy-proven metastasis or who had not had thorough conventional examination. In addition to these typical limitations, few studies stratified patients according to the different pathological types and metastatic locations. 
Table III. Stratification analysis of primary sites according to pathological type.

\begin{tabular}{|c|c|c|c|c|c|}
\hline Pathological type & Adenocarcinoma & $\begin{array}{l}\text { Squamous cell } \\
\text { carcinoma }\end{array}$ & $\begin{array}{l}\text { Small-cell } \\
\text { carcinoma }\end{array}$ & Other & Undefined \\
\hline $\begin{array}{l}\text { Primary sites (successful } \\
\text { detection/total number) }\end{array}$ & $38 / 179(21.2 \%)$ & $36 / 121(29.8 \%)$ & $3 / 26(11.5 \%)$ & $8 / 28(28.6 \%)$ & $29 / 76(38.2 \%)$ \\
\hline Lung & 15 & 6 & 2 & 3 & 14 \\
\hline Nasopharynx & 1 & 11 & 0 & 0 & 4 \\
\hline Pancreas & 8 & 1 & 0 & 1 & 3 \\
\hline Tonsil & 1 & 9 & 0 & 1 & 0 \\
\hline Small intestine & 3 & 0 & 1 & 0 & 3 \\
\hline Stomach & 2 & 1 & 0 & 2 & 1 \\
\hline Larynx & 0 & 2 & 0 & 0 & 2 \\
\hline Ovary & 4 & 0 & 0 & 0 & 0 \\
\hline Esophagus & 0 & 3 & 0 & 0 & 0 \\
\hline Colorectum & 0 & 1 & 0 & 0 & 2 \\
\hline Kidney & 1 & 0 & 0 & 1 & 0 \\
\hline Mammary gland (male) & 1 & 0 & 0 & 0 & 0 \\
\hline Prostate & 1 & 0 & 0 & 0 & 0 \\
\hline Penis & 0 & 1 & 0 & 0 & 0 \\
\hline Cholecyst & 0 & 1 & 0 & 0 & 0 \\
\hline Uterus & 1 & 0 & 0 & 0 & 0 \\
\hline
\end{tabular}

All patients in whom primaries were successfully detected by F-18 FDG PET/CT were stratified according to pathological type. Due to the extremely low detection rate of primary sites in CUP patients with metastatic melanoma, these results are not included in the table. Among the 19 CUP patients with metastatic melanoma, the primary site of only one patient was located by F-18 FDG PET/CT in the small intestine. F-18 FDG PET/CT, fluorine-18-2-fluoro-2-deoxy-D-glucose positron emission tomography/computed tomography; CUP, carcinoma of unknown primary.

Tianjin Cancer Institute and Hospital is one of the largest cancer centers in China. Between January 2006 and July 2015, a total of 30,063 patients received a F-18 FDG PET/CT scan in our Department of Molecular Imaging and Nuclear Medicine. In this study, qualifying individuals were selected from among 26,763 patients who had received a F-18 FDG PET/CT scan between January 2006 and October 2014 using strict criteria. The primary carcinoma was successfully identified in approximately a quarter of CUP patients using standard F-18 FDG PET/CT after conventional examination methods had failed. This number was slightly lower than expected from referring to previous studies. Previous studies had indicated that the most frequently identified primaries in CUP identified by F-18 FDG PET/CT were lung or head and neck carcinoma $(14,22)$. In this study, the F-18 FDG PET/CT scan revealed that the most common primary was the lung $(40 / 115,34.8 \%)$, followed by the nasopharynx, pancreas, tonsil, small intestine, stomach, larynx, ovary, esophagus, colorectum and kidney. Head and neck carcinoma, including primaries in the nasopharynx, tonsil and larynx $(31 / 115,27.0 \%)$, was still one of the most commonly occurring primaries among CUP patients. In addition, single cases were identified in the male mammary gland, prostate, penis, cholecyst and uterus. In contrast, 4,395 individuals exhibited biopsy-proven metastases initially and received F-18 FDG PET/CT prior to conventional examination. Of these patients, the primary sites of the majority (4064/4395, 92.5\%) were successfully located by F-18 FDG PET/CT, which were further confirmed by biopsy, other auxiliary examination or follow-up. Thus, considering its high detection efficiency, early application of F-18 FDG PET/CT should be encouraged for locating the primaries prior to the use of other conventional examination methods for patients with metastasis. For patients whose primaries cannot be located by conventional examination, F-18 FDG PET/CT may also be used as an auxiliary method, with an expected detection rate of $\sim 1 / 4$. In those patients whose primary sites were successfully located, the majority originated from the lung and the head and neck $(71 / 115,61.7 \%)$.

CUP patients with metastases in the head and neck were separately analyzed in certain previous studies $(13,23,24)$. CUP accounts for 2 to $9 \%$ of all patients with head and neck carcinoma (23). Patients may receive accurate surgery and radiation therapy, and benefit from the precise identification of primary carcinoma (13). In the present study, metastases initially presenting in the head and neck accounted for the majority of patients. The performance of F-18 FDG PET/CT in detecting primary carcinoma in this subgroup (29.1\%) was comparative with its potency across all CUP patients. Of the patients whose primary sites were located, the lung (30.3\%) and the head and neck (nasopharynx, tonsil and larynx) $(45.5 \%)$ were the most common primaries. In addition, we performed stratification analysis according to the various pathological types and locations (patient number more than 20). In CUP patients with squamous cell carcinoma 
Table IV. Stratification analysis of primary sites according to anatomical position of metastasis.

\begin{tabular}{|c|c|c|c|c|}
\hline Position of metastasis & Head and neck & Bone & Inguen & Cerebrum \\
\hline $\begin{array}{l}\text { Primary sites (successful } \\
\text { detection/total number) }\end{array}$ & $66 / 227(29.1 \%)$ & $19 / 49(38.8 \%)$ & $5 / 28(17.9 \%)$ & $5 / 22(22.7 \%)$ \\
\hline Lung & 20 & 12 & 0 & 3 \\
\hline Nasopharynx & 16 & 0 & 1 & 0 \\
\hline Pancreas & 4 & 1 & 0 & 0 \\
\hline Tonsil & 11 & 0 & 0 & 0 \\
\hline Small intestine & 2 & 1 & 1 & 1 \\
\hline Stomach & 3 & 2 & 0 & 0 \\
\hline Larynx & 3 & 0 & 1 & 0 \\
\hline Ovary & 1 & 0 & 1 & 0 \\
\hline Esophagus & 2 & 1 & 0 & 0 \\
\hline Colorectum & 1 & 0 & 0 & 1 \\
\hline Kidney & 1 & 1 & 0 & 0 \\
\hline Mammary gland (male) & 1 & 0 & 0 & 0 \\
\hline Prostate & 0 & 1 & 0 & 0 \\
\hline Penis & 0 & 0 & 1 & 0 \\
\hline Cholecyst & 1 & 0 & 0 & 0 \\
\hline
\end{tabular}

Patients n whom primaries were successfully detected by F-18 FDG PET/CT were stratified according to the anatomical position of metastasis. Subgroups with a patient number greater than 20 are listed, with the exception of patients with axillary metastasis. Among the 49 patients whose metastases were initially identified in the axilla, the primary sites of only two patients were identified by F-18 FDG PET/CT in the pancreas and small intestine. F-18 FDG PET/CT, fluorine-18-2-fluoro-2-deoxy-D-glucose positron emission tomography/computed tomography.

metastasis (30.6\%), adenocarcinoma metastasis $(20.7 \%)$, small-cell carcinoma metastasis $(11.5 \%)$, osseous metastasis (38.8\%), inguinal metastasis $(17.9 \%)$ and cerebral metastasis (22.7\%), we suggest that F-18 FDG PET/CT may be selectively applied clinically.

In the present study, we observed that F-18 FDG PET/CT had an extremely low detection rate in locating the primary carcinoma of metastatic melanoma and axillary metastasis in CUP patients. Melanoma derives from melanocytes, and is a type of lethal malignant disease (25). The survival rate of patients and their treatment strategy are closely related to the extent and stage of disease at the first visit to the clinic (26). However, large-sample studies have indicated that in $2-3 \%$ of melanoma cases, metastases were initially observed without detecting the primary carcinoma (27), which often led to difficulty in treatment. Particularly in patients with only metastatic lymph nodes or cutis, it is difficult to distinguish between distant and regional metastases, which is critical to the treatment strategy. F-18 FDG PET/CT is a useful tool and a particularly significant method in staging cutaneous and noncutaneous melanoma, respectively $(28,29)$. Also, although argument existed, it demonstrated a positive performance in monitoring relapse and judging the prognosis of cutaneous melanoma patients (29).

Currently, although F-18 FDG PET/CT has proven its potent diagnostic effect, only a few case reports have focused on its diagnostic role in metastatic melanoma of unknown primary. As far as we know, this study with a sample size of 19 metastatic melanoma patients is one of the largest studies on this topic. In this study, among the 19 patients with metastatic melanoma, the primary site (small intestine) of only one patient was identified by F-18 FDG PET/CT (1/19, 5.3\%). In addition, F-18 FDG PET/CT only led to treatment modification for one patient (canceled surgery), due to identification of further malignancy $(1 / 19,5.3 \%)$. Thus, considering the low detection rate, we do not recommend regular application of F-18 FDG $\mathrm{PET} / \mathrm{CT}$ for the sole purpose of locating the primary site in metastatic melanoma patients, if conventional methods have failed to identify this.

Clinically, malignant diseases that initially present with axillary metastasis are most commonly observed in breast malignancies (30). Due to the high diagnostic performance of mammography, breast ultrasonography and MRI $(31,32)$, the primaries of almost all patients with breast malignancies were detected initially, and so such patients were not included in our study. Thus, in the present study, CUP patients presenting with axillary metastasis usually had extramammary malignant diseases or occult breast malignancies that are difficult to detect with the commonly used methods. As far as we know, there is little relevant research in this field, with the exception of the study of Bertozzi et al (30). Based on our data, F-18 FDG PET/CT has an extremely low detection rate in CUP patients with axillary metastasis $(4.1 \%)$. As a result, we have serious concerns as to the clinical benefit of wide application of F-18 FDG PET/CT in detecting primary carcinoma of CUP patients with axillary metastasis in cases where conventional examination has failed to identify the primaries.

Previous studies have indicated that F-18 FDG PET/CT is able to detect primaries and extra metastases that were previously undetected, which leads to treatment strategy 
modification (14). According to previous data, approximately one-third of all patients (29.4 to $33.8 \%$ ) changed their treatment strategy, guided by F-18 FDG PET/CT $(12,14,16)$. This study was consistent with previous studies, and our data confirmed that F-18 FDG PET/CT led to treatment modification in $29 \%$ of all patients and $32.2 \%$ of head and neck metastasis patients. Thus, for the purpose of treatment instruction, we recommend active application of F-18 FDG PET/CT, and certain patients are likely to benefit from it.

In conclusion, to the best of our knowledge, the present study currently has the largest sample size in this field of research. F-18 FDG PET/CT has proven its clinical value in CUP patients. Its use is expected to identify the primaries in certain patients, and such patients are likely to benefit from its application. The most common primary sites appear at the lung and head and neck. Clinically, the use of F-18 FDG PET/CT should be encouraged earlier in CUP patients, or it should be applied as a complementary radiological method if conventional examination fails, except in cases of metastatic melanoma and in patients with axillary metastasis. For CUP patients with a pathological type of melanoma or a metastatic location in the axilla, we doubt the effectiveness and clinical benefit of the F-18 FDG PET/CT scan, in view of its extremely low positive rate.

\section{Acknowledgements}

This project was supported by grants from the National Natural Science Funds (81501984), Tianjin Municipal Bureau of Health Science and Technology Fund (2013KZ088), the National Science and Technology Major Project (2013ZX09303001) and the Health Bureau of Tianjin (2013KZ090).

\section{References}

1. Pavlidis N, Briasoulis E, Hainsworth J and Greco FA: Diagnostic and therapeutic management of cancer of an unknown primary. Eur J Cancer 39: 1990-2005, 2003.

2. Le Chevalier T, Cvitkovic E, Caille P, Harvey J, Contesso G, Spielmann M and Rouesse J: Early metastatic cancer of unknown primary origin at presentation. A clinical study of 302 consecutive autopsied patients. Arch Intern Med 148: 2035-2039, 1988.

3. Hainsworth JD, Spigel DR, Clark BL, Shipley D, Thompson DS, Farley C, West-Osterfield K, Lane CM, Cescon T, Bury MJ and Greco FA: Paclitaxel/carboplatin/etoposide versus gemcitabine/irinotecan in the first-line treatment of patients with carcinoma of unknown primary site: a randomized, phase III Sarah Cannon Oncology Research Consortium Trial. Cancer J 16: 70-75, 2010.

4. Møller AK, Pedersen KD, Gothelf A and Daugaard G: Paclitaxel, cisplatin and gemcitabine in treatment of carcinomas of unknown primary site, a phase II study. Acta Oncol 49: 423-430, 2010.

5. Fernandez-Cotarelo MJ, Guerra-Vales JM, Colina F and de la Cruz J: Prognostic factors in cancer of unknown primary site. Tumori 96: 111-116, 2010.

6. Hainsworth JD, Daugaard G, Lesimple T, Hübner G, Greco FA, Stahl MJ, Büschenfelde CM, Allouache D, Penel N, Knoblauch P and Fizazi KS: Paclitaxel/carboplatin with or without belinostat as empiric first-line treatment for patients with carcinoma of unknown primary site: a randomized, phase 2 trial. Cancer 121: 1654-1661, 2015.

7. Huang SW, Hsu CM, Jeng WJ, Yen TC, Su MY and Chiu CT: A comparison of positron emission tomography and colonoscopy for the detection of advanced colorectal neoplasms in subjects undergoing a health check-up. PLoS One 8: e69111, 2013.

8. Sun N, Zhao J, Qiao W and Wang T: Predictive value of interim PET/CT in DLBCL treated with R-CHOP: meta-analysis. Biomed Res Int 2015: 648572, 2015.
9. Lin J, Kligerman S, Goel R, Sajedi P, Suntharalingam M and Chuong MD: State-of-the-art molecular imaging in esophageal cancer management: implications for diagnosis, prognosis, and treatment. J Gastrointest Oncol 6: 3-19, 2015.

10. OS AA, Fischbein NJ, Caputo GR, Kaplan MJ, Price DC, Singer MI, Dillon WP and Hawkins RA: Metastatic head and neck cancer: role and usefulness of FDG PET in locating occult primary tumors. Radiology 210: 177-181, 1999.

11. Nassenstein K, Veit-Haibach P, Stergar H, Gutzeit A, Freudenberg L, Kuehl H, Fischer M, Barkhausen J, Bockisch A and Antoch G: Cervical lymph node metastases of unknown origin: primary tumor detection with whole-body positron emission tomography/computed tomography. Acta Radiol 48: 1101-1108, 2007.

12. Wang G, Wu Y, Zhang W, Li J, Wu P and Xie C: Clinical value of whole-body F-18 fluorodeoxyglucose positron emission tomography/computed tomography in patients with carcinoma of unknown primary. J Med Imaging Radiat Oncol 57: 65-71, 2013.

13. Lee JR, Kim JS, Roh JL, Lee JH, Baek JH, Cho KJ, Choi SH, Nam SY and Kim SY: Detection of occult primary tumors in patients with cervical metastases of unknown primary tumors: comparison of (18)F FDG PET/CT with contrast-enhanced CT or CT/MR imaging-prospective study. Radiology 274: 764-771, 2015.

14. Elboga U, Kervancioglu S, Sahin E, Basibuyuk M, Celen YZ and Aktolun C: Utility of F-18 fluorodeoxyglucose positron emission tomography/computed in carcinoma of unknown primary. Int J Clin Exp Pathol 7: 8941-8946, 2014.

15. Breuer N, Behrendt FF, Heinzel A, Mottaghy FM, Palmowski M and Verburg FA: Prognostic relevance of (18)F-FDG PET/CT in carcinoma of unknown primary. Clin Nucl Med 39: 131-135, 2014.

16. Hu M, Zhao W, Zhang PL, Ju GF, Fu Z, Zhang GL, Kong L, Yang YQ, Ma YD and Yu JM: Clinical applications of 18F-fluorodeoxyglucose positron emission tomography/computed tomography in carcinoma of unknown primary. Chin Med J (Engl) 124: 1010-1014, 2011.

17. Pak K, Kim SJ, Kim IJ, Nam HY, Kim BS, Kim K and Kim YK: Clinical implication of (18)F-FDG PET/CT in carcinoma of unknown primary. Neoplasma 58: 135-139, 2011

18. Yabuki K, Tsukuda M, Horiuchi C, Taguchi T and Nishimura G: Role of 18F-FDG PET in detecting primary site in the patient with primary unknown carcinoma. Eur Arch Otorhinolaryngol 267: 1785-1792, 2010.

19. Chorost MI, Lee MC, Yeoh CB, Molina M and Ghosh BC: Unknown primary. J Surg Oncol 87: 191-203, 2004.

20. Pelosi E, Pennone M, Deandreis D, Douroukas A, Mancini M and BisiG:Roleof wholebodypositronemissiontomography/computed tomography scan with $18 \mathrm{~F}$-fluorodeoxyglucose in patients with biopsy proven tumor metastases from unknown primary site. Q J Nucl Med Mol Imaging 50: 15-22, 2006.

21. Dong MJ, Zhao K, Lin XT, Zhao J, Ruan LX and Liu ZF: Role of fluorodeoxyglucose-PET versus fluorodeoxyglucose-PET/computed tomography in detection of unknown primary tumor: a meta-analysis of the literature. Nucl Med Commun 29: 791-802, 2008.

22. Wu ZJ, Zhang YX, Wei H and Jia Q: The role of whole body 2-[fluorine-18]-fluoro-2-deoxy-D-glucose positron emission tomography/computed tomography in the management of unknown primary tumors. Zhonghua Yi Xue Za Zhi 87: 2253-2256, 2007 (In Chinese).

23. Ryu IS, Choi SH, Kim do H, Han MW, Roh JL, Kim SY and Nam SY: Detection of the primary lesion in patients with cervical metastases from unknown primary tumors with narrow band imaging endoscopy: preliminary report. Head Neck 35: 10-14, 2013.

24. Zhao K, Luo XM, Zhou SH, Liu JH, Yan SX, Lu ZJ, Yang SY, Lin LL and Dong MJ: ${ }^{18} \mathrm{~F}$-fluorodeoxyglucose positron emission tomography/computed tomography as an effective diagnostic workup in cervical metastasis of carcinoma from an unknown primary tumor. Cancer Biother Radiopharm 27: 685-693, 2012.

25. de Vries E and Coebergh JW: Melanoma incidence has risen in Europe. BMJ 331: 698, 2005.

26. Rohren EM: PET/Computed tomography and patient outcomes in melanoma. PET Clin 10: 243-254, 2015.

27. Katz KA, Jonasch E, Hodi FS, Soiffer R, Kwitkiwski K, Sober AJ and Haluska FG: Melanoma of unknown primary: experience at Massachusetts General Hospital and Dana-Farber Cancer Institute. Melanoma Res 15: 77-82, 2005.

28. Krug B, Crott R, Lonneux M, Baurain JF, Pirson AS and Vander Borght T: Role of PET in the initial staging of cutaneous malignant melanoma: systematic review. Radiology 249: 836-844, 2008. 
29. Schwenzer NF and Pfannenberg AC: PET/CT, MR, and PET/MR in lymphoma and melanoma. Semin Nucl Med 45: 322-331, 2015.

30. Bertozzi S, Londero AP, Petri R and Bernardi S: Isolated axillary nodal swelling and cancer of unknown primary. Eur J Gynaecol Oncol 36: 131-137, 2015.

31. Tardivon AA, Athanasiou A, Thibault F and El Khoury C: Breast imaging and reporting data system (BIRADS) magnetic resonance imaging illustrated cases. Eur J Radiol 61: 216-223, 2007.
32. Mahoney MC, Gatsonis C, Hanna L, DeMartini WB and Lehman C: Positive predictive value of BI-RADS MR imaging. Radiology 264: 51-58, 2012. 